\title{
Unbounded Derivations and Invariant States
}

\author{
Ola Bratteli ${ }^{\star}$ \\ UER Scientifique de Marseille-Luminy, and Centre de Physique Theorique CNRS, \\ F-13274 Marseille Cedex 2, France \\ Uffe Haagerup \\ Odense Universitet, Matematisk Institut, DK-5230 Odense M, Denmark
}

\begin{abstract}
Let $\mathscr{M}$ be a von Neumann algebra with a cyclic and separating vector $\Omega$. Let $\delta=i[H, \cdot]$ be the spatial derivation implemented by a selfadjoint operator $H$, such that $H \Omega=0$. Let $\Delta$ be the modular operator associated with the pair $(\mathscr{M}, \Omega)$. We prove the equivalence of the following three conditions:

1) $H$ is essential selfadjoint on $D(\delta) \Omega$, and $H$ commutes strongly with $\Delta$.

2) The restriction of $H$ to $D(\delta) \Omega$ is essential selfadjoint on $D\left(\Delta^{\frac{1}{2}}\right)$ equipped with the inner product

$$
(\xi \mid \eta)_{\text {\#弄 }}=(\xi \mid \eta)+\left(\Delta^{\frac{1}{2}} \xi \mid \Delta^{\frac{1}{2}} \eta\right), \quad \xi, \eta \in D\left(\Delta^{\frac{1}{2}}\right) .
$$

3) $\exp (i t H) \mathscr{M} \exp (-i t H)=\mathscr{M}$ for any $t \in \mathbb{R}$.

We show by an example, that the first part of 1$), H$ is essential selfadjoint on $D(\delta) \Omega$, does not imply 3 ). This disproves a conjecture due to Bratteli and Robinson [3].
\end{abstract}

\section{Introduction}

In the study of time development of quantum and classical systems one often encounters the following situation ([5, 10,22] and references given there). The infinite volume Gibbs state $\omega$ is specified as a state on $C^{*}$-algebra $\mathfrak{A}$ of observables, along with a derivation $\delta$ of $\mathfrak{A}$, which should be the derivative at time zero for the time development of the infinite system. This derivation satisfies

$$
\omega \circ \delta(x)=0, \quad x \in D(\delta) .
$$

If $(\pi, \mathscr{H}, \Omega)$ is the cyclic representation associated to $\omega$, then there exists a unique symmetric operator $H_{0}$, satisfying the properties [3]:

(i) $D\left(H_{0}\right)=\pi(D(\delta)) \Omega$,

(ii) $H_{0} \Omega=0$,

(iii) $\pi(\delta(x)) \xi=i\left[H_{0}, \pi(x)\right] \xi, \xi \in D\left(H_{0}\right), x \in D(\delta)$,

* Part of this work was done while O.B. was a member of Zentrum für interdisziplinäre Forschung der Universität Bielefeld 
where $\left[H_{0}, x\right]=H_{0} x-x H_{0}$. The problem of proving the existence of a time development is therefore in a certain sense the problem of showing essential selfadjointness of $H_{0}$ on $\pi(D(\delta)) \Omega$, and to show that

$$
\exp (i t H) \mathscr{B} \exp (+i t H)=\mathscr{B}, \quad t \in \mathbb{R},
$$

where $\mathscr{B}$ is a suitable $C^{*}$-algebra containing $\pi(\mathfrak{U})$, and $H$ is the closure of $H_{0}$. In the best cases (free or "almost free" Fermi gases, spin systems with short range interactions $[21,24]), \mathscr{B}$ can be taken to be $\pi(\mathfrak{A})$ itself. In other cases (low density Bose- and Fermi gases, spin systems with long range interactions (cf. [5, 20]) one cannot expect $\pi(\mathfrak{U})$ itself to be globally invariant, but one can hope, and in some cases prove that the weak closure $\pi(\mathfrak{U})^{\prime \prime}$ is left invariant. Since the weak closure contains essentially the same observables as $\mathfrak{U}$ itself [12], this is a satisfactory solution to the problem of time development of the infinite system. Before proceeding we note that along with the existence of the derivation $\delta$ in the thermodynamic limit for the systems mentioned above, one also obtains uniform bounds for the derivatives of the approximate Greens functions for the system. These bounds imply that the vector $\Omega$ is separating for $\pi(\mathfrak{A})^{\prime \prime}$ (cf. [28], [5, Theorem 1]). Therefore, we will in the rest of the paper assume that $\Omega$ is both separating and cyclic for $\pi(\mathfrak{U})^{\prime \prime}$, and $\Delta$ will denote the modular operator associated with the pair $\left(\pi(\mathfrak{U})^{\prime \prime}, \Omega\right)$ (cf. [25]).

In this paper we prove that

$$
\exp (i t H) \pi(\mathfrak{U})^{\prime \prime} \exp (-i t H)=\pi(\mathfrak{U})^{\prime \prime}, \quad t \in \mathbb{R}
$$

under the following conditions :

a) $H$ is essentially selfadjoint on $\pi(D(\delta)) \Omega$,

b) $H$ commutes strongly with the modular operator $\Delta$ (i.e. all spectral projections commute).

The conditions are also necessary, and we prove by an example that in general a) does not imply b). This disproves a conjecture in [3]. In the case where $\Omega$ is a tracevector, the condition b) is, of course, empty so the present theorem is a generalization of the results in [4]. Other special cases of our theorem have been proved in $[3,6,10,14]$.

In a preliminary announcement of this paper, [7], a proof of the main theorem was outlined, whose main feature was to lift the derivation $\delta$ to the crossed product $R\left(\mathscr{M}, \sigma^{\omega}\right)$ of $\mathscr{M}=\pi(\mathfrak{H})^{\prime \prime}$ with the modular automorphism group $t \rightarrow \sigma_{t}^{\omega}$, utilizing $\sigma_{t}^{\omega} \circ \delta=\delta \circ \sigma_{t}^{\omega}$. The crossed product $R\left(\mathscr{M}, \sigma^{\omega}\right)$ is a semifinite von Neumann algebra and has a canonical, normal faithful semifinite trace $\tau,[27]$. One verifies that $\tau \circ \tilde{\delta}=0$, where $\tilde{\delta}$ is the lifted derivation, and then applies a generalization of the trace vector theorem in [4] to show that $\tilde{\delta}$ generates a group of automorphisms of $R\left(\mathscr{M}, \sigma^{\omega}\right)$. This group commutes with the dual action of $\hat{\mathbb{R}}=\mathbb{R}$ on $R\left(\mathscr{M}, \sigma^{\omega}\right)$, and since $\mathscr{M}$ is exactly the fixed points of the dual action, it follows that the automorphism group leaves $\mathscr{M}$ globally invariant, ending the proof of the theorem.

The method employed to prove the theorem in the present paper is less direct than suggested in the announcement, although based on the same idea. This is to avoid some technical difficulties associated with the treatment of the unbounded trace $\tau$. The present proof does not assume any a priori knowledge of the Connes- 
Takesaki duality theory, by introducing a third step in the proof. The proof is then as follows :

Step 1. If $\mathscr{M}_{\omega}=\left\{A \in \mathscr{M} \mid \sigma_{t}^{\omega}(A)=A, t \in \mathbb{R}\right\}$ is the centralizer for $\omega$, then

$$
\exp (i t H) \mathscr{M}_{\omega} \exp (-i t H)=\mathscr{M}_{\omega} .
$$

Step 2. If $\omega$ is periodic, i.e. $\sigma_{T}^{\omega}=1$ for some $T>0$, then $\exp (i t H) \mathscr{M} \exp (-i t H)=\mathscr{M}$. The proof is based on a decomposition of $\mathscr{M}$ in eigenspaces corresponding to $\sigma^{\omega}$ (as in [26]), and a $2 \times 2$ matrix trick employing Step 1 .

Step 3. Let $T>0$ and consider the discrete crossed product $\mathscr{N}=R\left(\mathscr{M}, \sigma_{T}^{\omega}\right)$. The dual weight $\tilde{\omega}$ of $\omega$ is then a state, and $\sigma_{T}^{\tilde{\omega}}$ is inner. By perturbing $\tilde{\omega}$ as in [8], we get a vector state $\omega^{\prime}$, such that $\sigma_{T}^{\omega^{\prime}}=1$, and $\omega^{\prime}(A)=\left(\Omega^{\prime} \mid A \Omega^{\prime}\right), A \in \mathcal{N}$, where $(H \otimes 1) \Omega^{\prime}=0$. Then Step 2 applies to prove that $\exp (i t(H \otimes 1)) \mathcal{N} \exp (-i t(H \otimes 1))=\mathscr{N}$, from which one can deduce that $\exp (i t H) \mathscr{M} \exp (-i t H)=\mathscr{M}$.

For application in quantum statistical mechanics it would be nice to have a version of our main theorem that can be applied when $\delta$ is a derivation from $D(\delta) \cong \mathscr{M}$ to certain unbounded operators affiliated with $\mathscr{M}$. In fact, such versions have been treated in [10] for abelian algebras, and in [4, p. 35] for algebras with an invariant tracial state. We have not tried to make such generalizations in our case, because it is difficult even to define such derivations in the general case, because when $\mathscr{M}$ is not finite, the set of closed, densily defined operator affiliated with $\mathscr{M}$ has no reasonable algebra structure $[17,23]$.

\section{§1. Preliminaries and Technicalities}

Let $H$ be a selfadjoint operator on a Hilbert space $\mathscr{H}$, and let

$$
\varrho_{t}(A)=\exp (i t H) A \exp (-i t H), \quad A \in \mathscr{L}(\mathscr{H}), \quad t \in \mathbb{R}
$$

be the corresponding one-parameter group of automorphisms of $\mathscr{L}(\mathscr{H})$. Denote by $\tilde{\delta}$ the infinitesimal generator of $\varrho$. Recall from [3, Theorem 4] that if $A \in \mathscr{L}(\mathscr{H})$, then the following conditions are equivalent

1) $A \in D(\tilde{\delta})$.

2) There exists a core $D$ for $H$, such that the sesquilinear form

$$
(\xi, \eta) \rightarrow i(H \xi \mid A \eta)-i(\xi \mid A H \eta)
$$

on $D \times D$ is bounded.

3) There exists a core $D$ for $H$, such that $A D \cong D$, and the mapping

$$
\xi \in D \rightarrow i[H, A] \xi
$$

is bounded.

If these conditions are satisfied, then the bounded operator associated with the sesquilinear form in 2) and the bounded mapping in 3 ) both coinside with $\tilde{\delta}(A)$.

If $\mathscr{M}$ is a von Neumann algebra on $\mathscr{H}$, we will in this paper use the terminology "the spatial derivation of $\mathscr{M}$ implemented by $H$ " to denote the restriction of $\tilde{\delta}$ to the domain

$$
D(\delta)=\{A \mid A \in D(\tilde{\delta}) \cap \mathscr{M}, \tilde{\delta}(A) \in \mathscr{M}\} .
$$


The map $\delta$ satisfies all the axioms for a symmetric derivation stated in [3], with the exception that the *algebra $D(\delta)$ is not necessarily $\sigma$-weakly dense in $\mathscr{M}$. The following lemma is essentially a restatement of results from [3], see also [16].

Lemma 1.1. Let $\delta=i[H, \cdot]$ be a spatial derivation of a von Neumann algebra $\mathscr{M}$ implemented by a selfadjoint operator $H$. Then

1) The ranges $R(1+\alpha \delta)$ are $\sigma$-weakly closed for each $\alpha \in \mathbb{R} \backslash\{0\}$.

2) The following conditions are equivalent

a) $R(1+\alpha \delta)=\mathscr{M}$ for some $\alpha>0$ and some $\alpha<0$.

b) $R(1+\alpha \delta)=\mathscr{M}$ for any $\alpha \in \mathbb{R} \backslash\{0\}$.

c) $\exp (i t H) \mathscr{M} \exp (-i t H)=\mathscr{M}$.

Proof. 1) Defining $\tilde{\delta}$ on $\mathscr{L}(\mathscr{H})$ as before the lemma, we have that $(1+\alpha \tilde{\delta})^{-1}$ exists as a $\sigma$-weakly continuous, bounded operator on $\mathscr{L}(\mathscr{H})$ for all $\alpha \in \mathbb{R} \backslash\{0\}([3$, Theorem 1]). But

$$
R(1+\alpha \delta)=\left\{A \in \mathscr{M} \mid(1+\alpha \tilde{\delta})^{-1} A \in \mathscr{M}\right\} .
$$

Hence $R(1+\alpha \delta)$ is $\sigma$-weakly closed.

2) c) $\Rightarrow$ a): Trivial from [3]. a) $\Rightarrow$ b): The condition $R(1+\alpha \delta)=\mathscr{M}$ is equivalent to $(1+\alpha \tilde{\delta})^{-1} \mathscr{M} \subseteq \mathscr{M}$. If this is true for some positive and some negative $\alpha$, it follows by successive applications of the Neumann expansion

$$
(1+\alpha \tilde{\delta})^{-1}=\frac{\alpha}{\alpha_{0}} \sum_{n \geqq 1}\left(\frac{\alpha-\alpha_{0}}{\alpha}\right)^{n-1}\left(1+\alpha_{0} \tilde{\delta}\right)^{-n}
$$

valid for $\left|\alpha-\alpha_{0}\right|<|\alpha|$ (cf. [15]), that the conditions is true for any $\alpha \in \mathbb{R} \backslash\{0\}$.

b) $\Rightarrow$ c) follows from the expansion

$$
\exp (i t H) A \exp (-i t H)=\lim _{n \rightarrow \infty}\left(1-\frac{t \tilde{\delta}}{n}\right)^{-n}(A)
$$

where the limit exists in the $\sigma$-weak topology (cf. [3]).

We say that two selfadjoint operators on a Hilbert space commute strongly if their spectral projections commute.

Lemma 1.2. Let $\mathscr{M}$ be a von Neumann algebra with a cyclic and separating vector $\Omega$, and let $\delta=i[H, \cdot]$ be the derivation implemented by a selfadjoint operator $H$, such that $H \Omega=0$, and $D(\delta) \Omega$ is a core for $H$. Let $\Delta$ be the modular operator associated with $\Omega$, and $\sigma_{t}^{\omega}$ the modular automorphism group associated with $\omega=(\Omega \mid \cdot \Omega)$. The following conditions are equivalent

1) $H$ commutes strongly with $\Delta$.

2) $\delta \circ \sigma_{t}^{\omega}=\sigma_{t}^{\omega} \circ \delta, t \in \mathbb{R}$.

Proof. 1) is equivalent to $H \Delta^{i t}=\Delta^{i t} H, t \in \mathbb{R}$, and hence 1$) \Rightarrow 2$ ) is an immediate consequence of the introductory remarks to this section. On the other hand 2) implies that $D(\delta)$ is globally invariant under $\sigma_{t}^{\omega}$, and hence $D(\delta) \Omega$ is invariant under $\Delta^{i t}$. Furthermore if $A \in D(\delta)$, then 2) implies that

$$
\begin{aligned}
i H \Delta^{i t} A \Omega & =\delta\left(\sigma_{t}^{\omega}(A)\right) \Omega \\
& =\sigma_{t}^{\omega}(\delta(A)) \Omega=\Delta^{i t} i H A \Omega .
\end{aligned}
$$


Since $D(\delta) \Omega$ is a core for $H$ it follows by closure that $H \Delta^{i t}=\Delta^{i t} H$.

The next lemma is a variant of Lemma 5 in [3].

Lemma 1.3. Let $\mathscr{M}$ be a von Neumann algebra with a cyclic and separating vector $\Omega$, and let $\delta=[i H, \cdot]$ be the spatial derivation implemented by a selfadjoint operator $H$, such that $H \Omega=0, D(\delta) \Omega$ is a core for $H$, and $H$ commutes strongly with the modular operator $\triangle$. Let $A, B \in \mathscr{M}$ and $\alpha \in \mathbb{R} \backslash\{0\}$. The following conditions are equivalent

1) $B \in D(\delta)$ and $A=(1+\alpha \delta)(B)$.

2) $(1+i \alpha H)^{-1} A \Omega=B \Omega$.

Proof. 1) $\Rightarrow 2$ ) is trivial. 2) $\Rightarrow 1$ ). Let $S$ be the modular conjugation operator, i.e. $S$ is the closure of $A \Omega \rightarrow A^{*} \Omega, A \in \mathscr{M}$, and let $S=J \Delta^{\frac{1}{2}}$ be its polar decomposition. As $\delta\left(A^{*}\right)=\delta(A)^{*}$, we deduce that

$$
i H J \Delta^{\frac{1}{2}} A \Omega=J \Delta^{\frac{1}{2}} i H A \Omega, \quad A \in D(\delta) .
$$

Using the fact that $H$ and $\Delta$ commute strongly, it follows that

$$
\Delta^{-\frac{1}{2}}[i H, J] A \Omega=0
$$

and hence

$$
[i H, J] A \Omega=0
$$

for all $A \Omega$ in the core $D(\delta) \Omega$ for $H$. By closure it follows that

$$
(i H) J=J(i H) \text {. }
$$

But then

$$
(1+i \alpha H)^{-1} J \Delta^{\frac{1}{2}} \cong J \Delta^{\frac{1}{2}}(1+i \alpha H)^{-1}
$$

and hence $(1+i \alpha H)^{-1} A \Omega=B \Omega$ implies that $(1+i \alpha H)^{-1} A^{*} \Omega=B^{*} \Omega$. The lemma now follows from the proof of Lemma 5 in [3].

\section{§2. Periodic States}

Let $\mathscr{M}$ be a von Neumann algebra on a Hilbert space $\mathscr{H}$ with a cyclic and separating vector $\Omega$, and let $\delta=i[H, \cdot]$ be the spatial derivation implemented by a selfadjoint operator $H$, such that $H \Omega=0, D(\delta) \Omega$ is a core for $H$, and $H$ commutes strongly with the modular operator $\Delta$ associated to $\Omega$. Put $\omega(A)=(\Omega \mid A \Omega), A \in \mathscr{M}$, and let $\sigma_{t}^{\omega}$ be the modular automorphism group associated with $\omega$. Recall from [25] that the centralizer $\mathscr{M}_{\omega}$ is the set of $A \in \mathscr{M}$, such that $\sigma_{t}^{\omega}(A)=A$ for all $t \in \mathbb{R}$, or equivalently the set of $A \in \mathscr{M}$, such that $\omega(A B)=\omega(B A)$ for all $B \in \mathscr{M}$.

Lemma 2.1. Let $\mathscr{M}_{\omega}$ be the centralizer for $\omega$. Then $\mathscr{M}_{\omega} \leqq R(1+\alpha \delta)$ for any $\alpha \in \mathbb{R} \backslash\{0\}$.

Proof. We start by proving that $\left(\mathscr{M}_{\omega} \cap R(1+\alpha \delta)\right) \Omega$ is dense in $\mathscr{M}_{\omega} \Omega$. Since $D(\delta) \Omega$ is a core for $H$, the spaces $R(1+\alpha \delta) \Omega=(1+i \alpha H) D(\delta) \Omega$ are dense in the Hilbertspace $\mathscr{H}$. Let $A \in \mathscr{M}_{\omega}$ and let $\varepsilon>0$. Choose $B \in R(1+\alpha \delta)$, so that $\|B \Omega-A \Omega\| \leqq \varepsilon$. Let $m$ be a left invariant mean on $\mathbb{R}$ (cf. [11]), and put

$$
B_{1}=\int_{-\infty}^{\infty} \sigma_{t}^{\omega}(B) d m(t), \quad(\sigma \text {-weakly })
$$


Clearly $\sigma_{t}^{\omega}\left(B_{1}\right)=B_{1}$ for any $t \in \mathbb{R}$, i.e. $B_{1}$ belongs to the centralizer $\mathscr{M}_{\omega}$. From Lemma 1.1 and 1.2 we have that $R(1+\alpha \delta)$ is $\sigma$-weakly closed, and invariant under $\sigma_{t}^{\omega}$. Hence $B_{1} \in R(1+\alpha \delta)$. Moreover

$$
\left\|\sigma_{t}^{\omega}(B) \Omega-A \Omega\right\|=\left\|\sigma_{t}^{\omega}(B-A) \Omega\right\|=\left\|\Delta^{i t}(B-A) \Omega\right\| \leqq \varepsilon .
$$

Now using that

$$
B_{1} \Omega=\int_{-\infty}^{\infty} \sigma_{t}^{\omega}(B) \Omega d m(t)
$$

in the weak topology on $\mathscr{H}$, we get that $\left\|B_{1} \Omega-A \Omega\right\| \leqq \varepsilon$. Hence $\left(R(1+\alpha \delta) \cap \mathscr{M}_{\omega}\right) \Omega$ is dense in $\mathscr{M}_{\omega}$.

Let now $A=A^{*} \in \mathscr{M}_{\omega}$. We can find a sequence $A_{n} \in R(1+\alpha \delta) \cap \mathscr{M}_{\omega}$ so that $\left\|A_{n} \Omega-A \Omega\right\| \rightarrow 0$. As $\Omega$ is a trace vector for $\mathscr{M}_{\omega}$ we have also $\left\|A_{n}^{*} \Omega-A \Omega\right\| \rightarrow 0$. Hence by replacing $A_{n}$ by $\frac{1}{2}\left(A_{n}+A_{n}^{*}\right)$ we get $A_{n}=A_{n}^{*} \in R(1+\alpha \delta)$ and $\left\|A_{n} \Omega-A \Omega\right\| \rightarrow 0$ for $n \rightarrow \infty$. Let $B_{n}=B_{n}^{*}$ be the operators in $D(\delta)$ for which $A_{n}=(1+\alpha \delta) B_{n}$. Then by Lemma $1.3 B_{n} \Omega=(1+i \alpha H)^{-1} A_{n} \Omega$. Since $\delta$ commutes with $\sigma_{t}^{\omega}$, and $A_{n} \in \mathscr{M}_{\omega}$, we have $B_{n} \Omega=\sigma_{t}^{\omega}\left(B_{1}\right) \Omega$. Since $\Omega$ is separating for $\mathscr{M}$, we have also $B_{n} \in \mathscr{M}_{\omega}$. Moreover $B_{n} \Omega \rightarrow(1+i \alpha H)^{-1} A \Omega$ for $n \rightarrow \infty$. Since $\Omega$ is a trace vector for $\mathscr{M}_{\omega}$, and since $\Omega$ is separating for $\mathscr{M}_{\omega}$, it follows from the proof of [4, Lemma 1$]$ that there exists a selfadjoint operator $B$ affiliated with $\mathscr{M}_{\omega}$, such that $\chi\left(B_{n}\right) \rightarrow \chi(B)$ strongly for any $C^{\infty}$-function $\chi$ on $\mathbb{R}$ with compact support, and such that

$$
B \Omega=\lim _{n \rightarrow \infty} B_{n} \Omega=(1+i \alpha H)^{-1} A \Omega .
$$

Now by the proof of $[4$, Lemma 2] we have

$$
\left(\Omega \mid \chi\left(B_{n}\right) B_{n} \Omega\right)=\left(\Omega \mid \chi\left(B_{n}\right) A_{n} \Omega\right) .
$$

Therefore in the limit $n \rightarrow \infty$ :

$$
(\Omega \mid \chi(B) B \Omega)=(\Omega \mid \chi(B) A \Omega) .
$$

It follows from this equation, that $B$ is bounded, and that $\|B\| \leqq\|A\|$. Assume namely that this was not the case. Then there exists a $C^{\infty}$-function $\chi \geqq 0$ with compact support, such that $\operatorname{supp}(\chi)$ is contained in $]-\infty,-\|A\|[$ or in $]\|A\|, \infty[$, and such that $\chi(B) \neq 0$. Using that $\Omega$ is a separating trace vector for $\mathscr{M}_{\omega}$ we get

but

$$
\begin{aligned}
|(\Omega \mid \chi(B) A \Omega)| & =\left|\left(\chi^{\frac{1}{2}}(B) \Omega \mid A \chi^{\frac{1}{2}}(B) \Omega\right)\right| \\
& \leqq\|A\|\left\|\chi^{\frac{1}{2}}(B) \Omega\right\|^{2} \\
& =\|A\|(\Omega \mid \chi(B) \Omega)
\end{aligned}
$$

$$
|(\Omega \mid \chi(B) B \Omega)|>(\Omega \mid\|A\| \chi(B) \Omega)=\|A\|(\Omega \mid \chi(B) \Omega)
$$

which is a contradiction. Hence $B \in \mathscr{M}_{\omega}$, and since $B \Omega=(1+i \alpha H)^{-1} A \Omega$ it follows from Lemma 1.3, that $B \in D(\delta)$ and that $A=(1+\alpha \delta) B$. This proves that any selfadjoint operator $A$ in $\mathscr{M}_{\omega}$ belongs to $R(1+\alpha \delta)$. Therefore $\mathscr{M}_{\omega} \cong R(1+\alpha \delta)$.

Lemma 2.2. Let $A \in \mathscr{M}$, and assume that there exists $\lambda>0$, such that $\sigma_{t}^{\omega}(A)=\lambda^{i t} A$ for any $t \in \mathbb{R}$, then $A \in R(1+\alpha \delta)$ for any $\alpha \in \mathbb{R} \backslash\{0\}$. 
Proof. Let $\mathrm{F}_{2}$ be the $I_{2}$-factor of $2 \times 2$-matrices acting on the Hilbert-space $\mathscr{H}_{2}$ of $2 \times 2$-matrices with Hilbert-Schmidt norm $\|x\|=\sum_{i, j}\left|x_{i j}\right|^{2}$. The von Neuman algebra $\mathscr{M} \otimes F_{2}$ acts on the Hilbertspace $\mathscr{H} \otimes \mathscr{H}_{2}$. Let $\left(e_{i j}\right)_{i, j=1,2}$ be the set of matrix units for $F_{2}$. Put

$$
\tilde{\Omega}=\Omega \otimes\left(e_{11}+\lambda^{\frac{1}{2}} e_{22}\right) \in \mathscr{H} \otimes \mathscr{H}_{2} .
$$

Then $\tilde{\Omega}$ is cyclic and separating for $\mathscr{M} \otimes F_{2}$, and the vector functional $\tilde{\omega}$ associated with $\tilde{\Omega}$ is given by

$$
\tilde{\omega}(B)=\omega\left(B_{11}+\lambda B_{22}\right), \quad B=\Sigma B_{i j} \otimes e_{i j} \in \mathscr{M} \otimes F_{2} .
$$

From [8, Chapter I] it follows that

$$
\sigma_{t}^{\tilde{\omega}}\left(B \otimes e_{12}\right)=(D \omega: D \lambda \omega)_{t} \sigma_{t}^{\lambda \omega}(B) \otimes e_{12}=\lambda^{-i t} \sigma_{t}^{\omega}(B) \otimes e_{12}
$$

for $B \in \mathscr{M}$. In particular when $\sigma_{t}^{\omega}(A)=\lambda^{i t} A$ we get $\sigma_{t}^{\omega}\left(A \otimes e_{12}\right)=A \otimes e_{12}$, i.e. $A \otimes e_{12}$ is in the centralizer for $\tilde{\omega}$. Let now $\tilde{\delta}$ be the derivation on $\mathscr{M} \otimes F_{2}$ implemented by $\tilde{H}=H \otimes 1$. Clearly

$$
D(\tilde{H})=\left\{x=\Sigma x_{i j} \otimes e_{i j} \mid x_{i j} \in D(H)\right\}
$$

and

$$
\tilde{H}\left(\Sigma x_{i j} \otimes e_{i j}\right)=\Sigma H x_{i j} \otimes e_{i j}, \quad \text { for } \quad x_{i j} \in D(H) .
$$

Moreover it is easily verified that

$$
D(\tilde{\delta})=\left\{B=\Sigma B_{i j} \otimes e_{i j} \mid B_{i j} \in D(\delta)\right\}
$$

and

$$
\tilde{\delta}\left(\Sigma B_{i j} \otimes e_{i j}\right)=\Sigma \delta\left(B_{i j}\right) \otimes e_{i j}, \quad B_{i j} \in D(\delta) .
$$

Clearly $\tilde{\Omega} \in D(\tilde{H})$, and $\tilde{H} \tilde{\Omega}=0$. Moreover,

$$
D(\tilde{\delta}) \tilde{\Omega}=D(\delta) \Omega \otimes \mathscr{H}_{2}
$$

which proves that $D(\tilde{\delta}) \tilde{\Omega}$ is a core for $\tilde{H}$. By $[8$, Chapter I] we get that

$$
\sigma_{t}^{\tilde{\omega}}\left(\begin{array}{ll}
B_{11} & B_{12} \\
B_{21} & B_{22}
\end{array}\right)=\left(\begin{array}{cc}
\sigma_{t}^{\omega}\left(B_{11}\right) & \lambda^{-i t} \sigma_{t}^{\omega}\left(B_{12}\right) \\
\lambda^{i t} \sigma_{t}^{\omega}\left(B_{21}\right) & \sigma_{t}^{\omega}\left(B_{22}\right)
\end{array}\right)
$$

for $B=\Sigma B_{i j} \otimes e_{i j} \in \mathscr{M} \otimes F_{2}$.

Since $\delta$ commutes with $\sigma_{t}^{\omega}$, it follows that $\tilde{\delta}$ is $\sigma_{t}^{\tilde{\omega}}$-invariant. Hence by Lemma $1.2 \tilde{H}$ commutes strongly with the modular operator $\tilde{\Delta}$ associated with $\tilde{\Omega}$. Therefore $\mathscr{M} \otimes F_{2}, \tilde{\Omega}$ and $\tilde{\delta}=i[\tilde{H}, \cdot]$ satisfy the conditions of Lemma 2.1 . Since $A \otimes e_{12}$ is in the centralizer of $\sigma_{t}^{\tilde{\omega}}$ it follows that $A \otimes e_{12} \in R(1+\alpha \tilde{\delta})$ or equivalently $A \in R(1+\alpha \delta), \alpha \in \mathbb{R} \backslash\{0\}$.

Recall that the functional $\omega(A)=(\Omega \mid A \Omega)$ is called periodic if $\sigma_{T}^{\omega}=1$ for some non zero $T \in \mathbb{R}$.

Proposition 2.3. Let $\mathscr{M}, \Omega, H$, and $\delta$ be as in the beginning of $\S 2$, and assume that the functional $\omega(A)=(\Omega \mid A \Omega)$ is periodic. Then

$$
\exp (i t H) \mathscr{M} \exp (-i t H)=\mathscr{M}, \quad t \in \mathbb{R} .
$$


Proof. By the assumptions $\Delta^{i T}=1$ for some $T>0$ or equivalently $\sigma_{T}^{\omega}=1$. Put $k=e^{-2 \pi / T}$. Since $\sigma^{\omega}$ is periodic, the eigenspaces for $\sigma^{\omega}$,

$$
\mathscr{M}_{n}=\left\{A \in \mathscr{M} \mid \sigma_{t}^{\omega}(A)=k^{\mathrm{int}} A\right\}, \quad n \in \mathbb{Z}
$$

span a $\sigma$-weakly dense subalgebra of $\mathscr{M}$ (cf. $[1,26]$ ). By Lemma 2.2 $R(1+\alpha \delta) \supseteqq \bigcup_{n \in \mathbb{Z}} \mathscr{M}_{n}$. Hence by Lemma $1.1 R(1+\alpha \delta)=\mathscr{M}$ for any $\alpha \in \mathbb{R} \backslash\{0\}$ and consequently $\exp (i t H) \mathscr{M} \exp (-i t H)=\mathscr{M}, t \in \mathbb{R}$.

For the proof of the main theorem in $\S 3$ we need a slight generalization of Proposition 2.3.

Corollary 2.4. Let $\mathscr{M}, \Omega, H, \delta$ satisfy the assumptions in the beginning of $\S 2$, and let $T>0$. Assume that there exists a unitary operator $U \in \mathscr{M}$, so that

$$
\sigma_{T}^{\omega}(A)=U A U^{*}, \quad A \in \mathscr{M}, \text { and }[H, U]=0
$$

then

$$
\exp (i t H) \mathscr{M} \exp (-i t H)=\mathscr{M} \text {. }
$$

Proof. Since $\omega\left(U A U^{*}\right)=\omega\left(\sigma_{T}^{\omega}(A)\right)=\omega(A)$ for $A \in \mathscr{M}$, it follows that $U$ belongs to the centralizer of $\omega$ (cf. [8, Proof of Theorem 1.3.2]). Put $A=-i \log (U)$, where $\log$ is the branch of the logarithm function for which $-\pi<\operatorname{Im}(\log (a)) \leqq \pi$. Then $A=A^{*}$ and $\|A\| \leqq \pi$. Put $B=\exp \left(\frac{1}{T} A\right)$. Then $B$ is positive selfadjoint with bounded inverse, $B^{i T}=U$ and $B \in \mathscr{M}_{\omega}$. Moreover as $[H, U]=0$ we have $\left[H, B^{\alpha}\right]=0$ $\forall \alpha \in \mathbb{C}$. In particular $B^{\alpha} \in D(\delta)$. Put $\Omega^{\prime}=B^{-\frac{1}{2}} \Omega$. We will prove that $\mathscr{M}, \Omega^{\prime}, H$, and $\delta$ satisfy the conditions of Proposition 2.3:

Since $B^{\frac{1}{2}}$ and $B^{-\frac{1}{2}}$ are bounded we have that $\Omega^{\prime}=B^{-\frac{1}{2}} \Omega$ is cyclic and separating for $\mathscr{M}$. Put $\omega^{\prime}(A)=\left(\Omega^{\prime} \mid A \Omega^{\prime}\right)$.

Then

$$
\omega^{\prime}(A)=\omega\left(B^{-\frac{1}{2}} A B^{-\frac{1}{2}}\right)=\omega\left(B^{-1} A\right), \quad A \in \mathscr{M},
$$

because $B^{-\frac{1}{2}} \in \mathscr{M}_{\omega}$. Hence by [19]

$$
\sigma_{T}^{\omega^{\prime}}(A)=B^{-i T} \sigma_{T}^{\omega}(A) B^{i T}=U^{*}\left(U A U^{*}\right) U=A,
$$

i.e. $\omega^{\prime}$ is a periodic state. Since $B^{\frac{1}{2}}, B^{-\frac{1}{2}} \in D(\delta)$ we have $D(\delta) \Omega^{\prime}=D(\delta) \Omega$. Therefore $D(\delta) \Omega^{\prime}$ is a core for $H$. Let $\Delta^{\prime}$ be the modular operator associated with $\Omega^{\prime}$. To prove that $H$ and $\Delta^{\prime}$ commute strongly, it is enough to prove that $\delta$ is $\sigma_{t}^{\omega^{\prime}}$-invariant (cf. Lemma 1.2). However $\sigma_{t}^{\omega^{\prime}}(A)=B^{-i t} \sigma_{t}^{\omega}(A) B^{+i t}$, and since $\delta$ commutes with $\sigma_{t}^{\omega}$ and $\delta\left(B^{i t}\right)=\delta\left(B^{-i t}\right)=0$ the assertion follows.

Hence by Proposition 2.3:

$$
\exp (i t H) \mathscr{M} \exp (-i t H)=\mathscr{M}, \quad t \in \mathbb{R} \text {. }
$$

\section{§3. General Faithful States}

We are now ready to prove the main theorem of this paper. In the end of this section we will derive an alternative way of stating Condition 1) in terms of the graph Hilbert norm associated to $\Delta^{\frac{1}{2}}$, and in $\S 4$ we show by an example, that the 
condition that $H$ and $\Delta$ commute strongly cannot be excluded from the main theorem (Theorem 3.1).

Theorem 3.1. Let $\mathscr{M}$ be a von Neumann algebra on a Hilbert space $\mathscr{H}$ with a cyclic and separating vector $\Omega$, and let $\delta=i[H, \cdot]$ be the spatial derivation on $\mathscr{M}$ implemented by a selfadjoint operator $H$, such that $H \Omega=0$. The following conditions are equivalent

1) $D(\delta) \Omega$ is a core for $H$, and $H$ commutes strongly with the modular operator $\Delta$ associated with $\Omega$.

2) $\exp (i t H) \mathscr{M} \exp (-i t H)=\mathscr{M} \forall t \in \mathbb{R}$.

Proof. 2) $\Rightarrow 1)$. If 2) is satisfied it follows from Lemma 1.1 that $(1+i \alpha H) D(\delta) \Omega$ $=R(1+i \alpha \delta) \Omega=\mathscr{M} \Omega$ for $\alpha \in \mathbb{R} \backslash\{0\}$. Thus $D(\delta) \Omega$ is a core for $H$. Put $\alpha_{t}(A)=e^{i t H} A e^{-i t H}, A \in \mathscr{M}, t \in \mathbb{R}$. Put $\omega=(\Omega \mid \cdot \Omega)$. Since $\exp (i t H) \Omega=\Omega$ we have $\omega\left(\alpha_{t}(A)\right)=\omega(A)$, and by the uniqueness of the modular automorphism group

$$
\sigma_{s}^{\omega} \circ \alpha_{t}=\alpha_{t} \circ \sigma_{s}^{\omega}, \quad s, t \in \mathbb{R} .
$$

Thus $\Delta^{i s} e^{i t H} A \Omega=e^{i t H} \Delta^{i s} A \Omega$ for $A \in \mathscr{M}$ and hence $\Delta^{i s} e^{i t H}=e^{i t H} \Delta^{i s}$, i.e. $\Delta$ and $H$ commute strongly.

$1) \Rightarrow 2$ ). Let $T>0$ be a fixed real number. Consider the discrete crossed product $\mathscr{N}=R\left(\mathscr{M}, \sigma_{T}^{\omega}\right)$. Let $\tilde{\mathscr{H}}$ be the Hilbert space

$$
\tilde{\mathscr{H}}=\sum_{n=-\infty}^{\infty} \mathscr{H}_{n},
$$

where $\mathscr{H}_{n}=\mathscr{H}, n \in \mathbb{Z}$. The von Neumann algebra $\mathscr{N}$ is generated by the operators $\pi(A)$ defined by

$$
(\pi(A) \xi)_{n}=\sigma_{-n T}^{\omega}(A) \xi_{n}, \quad A \in \mathscr{M}, \quad \xi=\left(\xi_{n}\right)_{n} \in \tilde{\mathscr{H}}
$$

and the unitary operator $U$ given by

$$
(U \xi)_{n}=\xi_{n-1}, \quad\left(\xi_{n}\right)_{n} \in \tilde{\mathscr{H}} .
$$

Note that $U \pi(A) U^{*}=\pi\left(\sigma_{T}^{\omega}(A)\right), A \in \mathscr{M}$.

Consider the vector $\tilde{\Omega} \in \mathscr{\mathscr { H }}$ given by

$$
\tilde{\Omega}_{n}= \begin{cases}\Omega & n=0 \\ 0 & n \neq 0\end{cases}
$$

and let $\tilde{\omega}$ be the positive functional on $\mathscr{N}$ given by

$$
\tilde{\omega}(A)=(\tilde{\Omega} \mid A \tilde{\Omega}), \quad A \in \mathscr{N} .
$$

For the rest of the proof we need a lemma:

Lemma 3.2. 1) $\tilde{\Omega}$ is cyclic and separating for $\mathscr{N}$.

2) The modular automorphism group associated with $\tilde{\omega}$ is given by

$$
\begin{aligned}
\sigma_{t}^{\tilde{\omega}}(\pi(A)) & =\pi\left(\sigma_{t}^{\omega}(A)\right), \quad A \in \mathscr{M} . \\
\sigma_{t}^{\omega}(U) & =U, \quad t \in \mathbb{R} . \\
\text { 3) } \sigma_{T}^{\tilde{\omega}}(B) & =U B U^{*}, \quad B \in \mathscr{N} .
\end{aligned}
$$


4) The modular operator $\tilde{\Delta}$ associated with $\tilde{\Omega}$ is given by

$$
\left(\tilde{\Delta}^{i t} \xi\right)_{n}=\Delta^{i t} \xi_{n}, \quad\left(\xi_{n}\right)_{n} \in \tilde{\mathscr{H}} .
$$

Proof. Lemma 3.2 is in fact just a very special case of the general theory of dual weights for crossed products cf. $[9,13]$ but for convenience of the reader we give a selfcontained proof.

1) Let $\mathscr{N}_{0}$ be the $\sigma$-weakly dense subalgebra of $\mathscr{N}$ generated by $\pi(\mathscr{M})$ and $U$. We have

$$
\mathscr{N}_{0}=\left\{A=\sum_{n=-p}^{p} U^{n} \pi\left(A_{n}\right) \mid p \in \mathbb{N}, A_{n} \in \mathscr{M}\right\} .
$$

Since the maps $A \rightarrow A_{n}, A \in \mathscr{N}_{0}$ are $\sigma$-strongly continuous, any element $A \in \mathscr{N}$ has an expansion

$$
A \sim \sum_{n=-\infty}^{\infty} U^{n} \pi\left(A_{n}\right), \quad A_{n} \in \mathscr{M}
$$

which converges in the sense that for all $\xi \in \tilde{\mathscr{H}}$ with finite support

$$
A \xi=\sum_{n=-\infty}^{\infty} U^{n} \pi\left(A_{n}\right) \xi
$$

In particular

$$
A \tilde{\Omega}=\sum_{n=-\infty}^{\infty} U^{n} \pi\left(A_{n}\right) \tilde{\Omega} .
$$

Hence the $n$-te coordinate is $(A \tilde{\Omega})_{n}=A_{n} \Omega$.

Therefore $\|A \tilde{\Omega}\|^{2}=\sum_{n=-\infty}^{\infty}\left\|A_{n} \Omega\right\|^{2}$.

This proves that $\tilde{\Omega}$ is separating for $\mathscr{N}$. Clearly $\mathscr{N} \tilde{\Omega}$ contains all vectors $\left(\xi_{n}\right)_{n} \in \tilde{\mathscr{H}}$ with finite support, for which $\xi_{n} \in \mathscr{M} \Omega$. Hence $\tilde{\Omega}$ is also cyclic for $\mathscr{N}$.

2) Let $V_{t}$ be the one parameter unitary group on $\tilde{\mathscr{H}}$, given by $\left(V_{t} \xi\right)_{n}=\Delta^{i t} \xi_{n}$. It is trivial to check that

$$
\begin{aligned}
V_{t} \pi(A) V_{t}^{*} & =\pi\left(\sigma_{t}^{\omega}(A)\right), \quad A \in \mathscr{M} \\
V_{t} U V_{t}^{*} & =U .
\end{aligned}
$$

Hence $V_{t}$ implements a one parameter automorphism group $\sigma_{t}$ of $\mathscr{N}$. We will prove that $\tilde{\omega}$ is K.M.S. with respect to $\sigma_{t}$. For $A \in \mathscr{N}$, we have

$$
\begin{aligned}
& A \sim \sum_{n=-\infty}^{\infty} U^{n} \pi\left(A_{n}\right), \quad A^{*} \sim \sum_{n=-\infty}^{\infty} U^{n} \pi\left(\sigma_{-n T}^{\omega}\left(A_{-n}^{*}\right)\right) \\
& \sigma_{t}(A) \sim \sum_{n=-\infty}^{\infty} U^{n} \pi\left(\sigma_{t}^{\omega}\left(A_{n}\right)\right), \quad \sigma_{t}\left(A^{*}\right) \sim \sum_{n=-\infty}^{\infty} U^{n} \pi\left(\sigma_{t-n T}^{\omega}\left(A_{-n}^{*}\right)\right) .
\end{aligned}
$$


Hence for $A, B \in \mathscr{N}$ :

$$
\begin{aligned}
\tilde{\omega}\left(\sigma_{t}(A) B\right) & =\left(\sigma_{t}(A)^{*} \tilde{\Omega} \mid B \tilde{\Omega}\right) \\
& =\sum_{n=-\infty}^{\infty}\left(\sigma_{t-n T}^{\omega} A_{-n}^{*} \Omega \mid B_{n} \Omega\right) \\
& =\sum_{n=-\infty}^{\infty} \omega\left(\sigma_{t-n T}^{\omega}\left(A_{-n}\right) B_{n}\right)=\sum_{n=-\infty}^{\infty} \omega\left(\sigma_{t+n T}^{\omega}\left(A_{n}\right) B_{-n}\right) .
\end{aligned}
$$

A similar calculation gives

$$
\begin{aligned}
\tilde{\omega}\left(B \sigma_{t}(A)\right) & =\left(B^{*} \tilde{\Omega} \mid \sigma_{t}(A) \tilde{\Omega}\right) \\
& =\sum_{n=-\infty}^{\infty} \omega\left(\sigma_{-n T}^{\omega}\left(B_{-n}\right) \sigma_{t}^{\omega}\left(A_{n}\right)\right) \\
& =\sum_{n=-\infty}^{\infty} \omega\left(B_{-n} \sigma_{t+n T}^{\omega}\left(A_{n}\right)\right),
\end{aligned}
$$

where we have used that $\omega$ is $\sigma_{T}^{\omega}$-invariant. Since $\omega$ is $\sigma_{t}^{\omega}$-K.M.S. and since one easily verifies that the above sums converge uniformly in $t$, it follows that $\tilde{\omega}$ is $\sigma_{t}-$ K.M.S. Hence by $[25, \S 14]$ we have $\sigma_{t}=\sigma_{t}^{\tilde{\omega}}$.

3) For $A \in \mathscr{M}$ we have by (2) that

$$
\sigma_{T}^{\tilde{\omega}}(\pi(A))=\pi\left(\sigma_{T}^{\omega}(A)\right)=U \pi(A) U^{*} .
$$

Moreover

$$
\sigma_{T}^{\tilde{\omega}}(U)=U=U U U^{*} .
$$

Hence

$$
\sigma_{T}^{\tilde{\omega}}(B)=U B U^{*} \quad \text { for any } \quad B \in \mathscr{N} .
$$

4) Let $A \in \mathscr{N}, A \sim \sum_{n=-\infty}^{\infty} U^{n} \pi\left(A_{n}\right)$.

We have

$$
(A \tilde{\Omega})_{n}=A_{n} \Omega
$$

and

$$
\left(\sigma_{t}^{\tilde{\omega}}(A) \tilde{\Omega}\right)_{n}=\sigma_{t}^{\omega}\left(A_{n}\right) \Omega .
$$

Since

$$
\tilde{\Delta}^{i t} A \tilde{\Omega}=\tilde{\Delta}^{i t} A \tilde{\Delta}^{-i t} \tilde{\Omega}=\sigma_{t}^{\tilde{\omega}}(A) \tilde{\Omega}
$$

we get for $\xi=\left(A_{n} \Omega\right)_{n} \in \tilde{\mathscr{H}}$, that

$$
\left(\tilde{\Delta}^{i t} \xi\right)_{n}=\sigma_{t}^{\omega} A_{n} \Omega=\Delta^{i t} A_{n} \Omega=\Delta^{i t} \xi_{n} .
$$

Since $\mathscr{N} \tilde{\Omega}$ is dense in $\tilde{\mathscr{H}}$, the assertion follows. 
End of Proof 1) $\Rightarrow 2$ ). Define a selfadjoint operator $\tilde{H}$ on $\tilde{\mathscr{H}}$ by

$$
D(\tilde{H})=\left\{\left(\xi_{n}\right)_{n} \in \tilde{\mathscr{H}} \mid \xi_{n} \in D(H) \text { and } \sum_{n=-\infty}^{\infty}\left\|H \xi_{n}\right\|^{2}<\infty\right\}
$$

and put $(\tilde{H} \xi)_{n}=H \xi_{n}$ for $\xi=\left(\xi_{n}\right)_{n} \in D(\tilde{H})$.

Let $\tilde{\delta}=i[\tilde{H}, \cdot]$ be the derivation on $\mathscr{N}$ implemented by $\tilde{H}$. We shall prove that $\mathscr{N}, \tilde{\Omega}, \tilde{H}, \tilde{\delta}$, and $U$ satisfy the conditions of Corollary 2.4 . Clearly $\tilde{H} \tilde{\Omega}=0$. Since $\delta \circ \sigma_{T}^{\omega}=\sigma_{T}^{\omega} \circ \delta$ one has that $A \in D(\delta)$ implies $\pi(A) \in D(\tilde{\delta})$ and $\tilde{\delta}(\pi(A))=\pi(\delta(A))$. Moreover $U \tilde{H}=\tilde{H} U$. Hence $U \in D(\delta)$ and $\delta(U)=0$. Therefore $D(\tilde{\delta})$ contains all operators of the form

$$
\sum_{n=-p}^{p} U^{n} \pi\left(A_{n}\right) \quad \text { where } A_{n} \in D(\delta) .
$$

In particular $D(\tilde{\delta}) \tilde{\Omega}$ contains all vectors $\left(\xi_{n}\right)_{n} \in \tilde{\mathscr{H}}$ of finite support for which $\xi_{n} \in D(\delta) \Omega$. Hence $D(\tilde{\delta}) \tilde{\Omega}$ is a core for $\tilde{H}$. Since $H$ commutes with $\Delta^{i t}$, we get by Lemma 3.2.4), that $\tilde{H}$ commutes with $\tilde{\Delta}^{i t}$. As $\sigma_{T}^{\tilde{\omega}}$ is implemented by $U$ and $[\tilde{H}, U]=0$, Corollary 2.4 can be applied. Hence

$$
\exp (i t \tilde{H}) \mathscr{N} \exp (-i t \tilde{H})=\mathscr{N}, \quad t \in \mathbb{R} .
$$
Now let $P$ be the projection from $\tilde{\mathscr{H}}=\sum_{n=-\infty}^{\infty} \mathscr{H}_{n}$ onto the zeroth component.
$P\left(\left(\xi_{n}\right)_{n}\right)=\xi_{0} \in \mathscr{H}_{0}=\mathscr{H}$.

Then for $A \in \mathscr{N}, A \sim \sum_{n=-\infty}^{\infty} U^{n} \pi\left(A_{n}\right)$ we have

$$
P A P^{*}=A_{0} .
$$

Moreover

$$
\exp (i t H) P=P \exp (i t \tilde{H}) .
$$

Let now $A \in \mathscr{N}$. Put $B=\exp (i t \tilde{H}) \pi(A) \exp (-i t \tilde{H}) \in \mathscr{N}$ and let

$$
B \sim \sum_{n=-\infty}^{\infty} U^{n} \pi\left(B_{n}\right), \quad B_{n} \in \mathscr{M}
$$

be the expansion of $B$. Then

$$
\begin{aligned}
\exp (i t H) A \exp (-i t H) & =\exp (i t H) P \pi(A) P^{*} \exp (-i t H) \\
& =P \exp (i t \tilde{H}) \pi(A) \exp (-i t \tilde{H}) P^{*} \\
& =P B P^{*}=B_{0} \in \mathscr{M} .
\end{aligned}
$$

Hence $\exp (i t H) \mathscr{M} \exp (-i t H) \subseteq \mathscr{M}$ for any $t \in \mathbb{R}$, ending the proof of the theorem.

In the last part of this section we will derive an alternative formulation of Theorem 3.1. This will be in terms of the graph Hilbert space $\mathscr{H}_{\#}$ of the square root of the modular operator $\Delta$ associated with $\Omega$, i.e. $\mathscr{H}_{\#}$ is the linear space $D\left(\Delta^{\frac{1}{2}}\right)$ equipped with the inner product

$$
(\xi \mid \eta)_{\text {\# }}=(\xi \mid \eta)+\left(\Delta^{\frac{1}{2}} \xi \mid \Delta^{\frac{1}{2}} \eta\right) \quad \xi, \eta \in \mathscr{H}_{\text {\# }} .
$$


Lemma 3.3. Let $\mathscr{M}$ be a von Neumann algebra with a cyclic and separating vector $\Omega$, and let $\delta=i[\mathrm{H}, \cdot \cdot]$ be the spatial derivation on $\mathscr{M}$ implemented by a selfadjoint operator $H$, such that $H \Omega=0$. It follows that

a) $D(\delta) \Omega \subseteq \mathscr{H}_{\#}$ and $H D(\delta) \Omega \subseteq \mathscr{H}_{\text {\# }}$.

b) The restriction of $H$ to $D(\delta) \Omega$ is symmetric with respect to the inner product $(\cdot \mid \cdot)_{\#} \cdot$

Proof. a) Clearly

$$
D(\delta) \Omega \subseteq \mathscr{M} \Omega \subseteq D\left(\Delta^{\frac{1}{2}}\right)=\mathscr{H}_{\text {\# }},
$$

and

$i H D(\delta) \Omega=R(\delta) \Omega \subseteq \mathscr{M} \Omega \cong \mathscr{H}_{\text {\#. }}$

b) Let $S=J \Delta^{\frac{1}{2}}$ be the modular involution associated with $\Omega$. Since $\delta\left(A^{*}\right)=\delta(A)^{*}, A \in D(\delta)$, we get for $A \in D(\delta)$ :

$$
\begin{aligned}
H S A \Omega=H A^{*} \Omega & =-i \delta\left(A^{*}\right) \Omega \\
& =-i \delta(A)^{*} \Omega=-S H A \Omega .
\end{aligned}
$$

Hence for $\xi, \eta \in D(\delta) \Omega$ we get

$$
\begin{aligned}
(\xi \mid H \eta)_{\#} & =(\xi \mid H \eta)+(S H \eta \mid S \xi) \\
& =(\xi \mid H \eta)-(H S \eta \mid S \xi) \\
& =(H \xi \mid \eta)-(S \eta \mid H S \xi) \\
& =(H \xi \mid \eta)+(S \eta \mid S H \xi)=(H \xi \mid \eta)_{\#} .
\end{aligned}
$$

Therefore the restriction of $H$ to $D(\delta) \Omega$ is symmetric on $\mathscr{H}_{\#}$.

Lemma 3.3 implies that the first statement of the following proposition makes sense.

Proposition 3.4. Let $\mathscr{M}$ be a von Neumann algebra on a Hilbert space $\mathscr{H}$ with a cyclic and separating vector $\Omega$, and let $\delta=i[H, \cdot]$ be the spatial derivation on $\mathscr{M}$ implemented by a selfadjoint operator $H$, such that $H \Omega=0$. The following conditions are equivalent.

1) The restriction of $H$ to $D(\delta) \Omega$ is essential selfadjoint as an operator on $\mathscr{H}_{\#}$.

2) $\exp (i t H) \mathscr{M} \exp (-i t H)=\mathscr{M}$.

Proof. 2) $\Rightarrow 1)$. By Lemma 1.1, Condition 2) implies that $(1+i \alpha H) D(\delta) \Omega$ $=R(1+\alpha \delta) \Omega=\mathscr{M} \Omega$ for $\alpha \in \mathbb{R} \backslash\{0\}$. But $\mathscr{M} \Omega$ is a core for $\Delta^{\frac{1}{2}}$, i.e. $\mathscr{M} \Omega$ is dense in $\mathscr{H}_{\#}$. As the restriction of $H$ to $D(\delta) \Omega$ is symmetric on $\mathscr{H}_{\#}$ by Lemma 3.3, it follows that $H \mid D(\delta) \Omega$ is essential selfadjoint as an operator on $\mathscr{H}_{\text {\# }}$.

$1) \Rightarrow 2$ ). It is enough to prove that 1) implies Condition 1) in Theorem 3.1. By 1) $(1+i \alpha H) D(\delta) \Omega$ is dense in $\mathscr{H}_{\#}$ for $\alpha \in \mathbb{R} \backslash\{0\}$ but then $(1+i \alpha H) D(\delta) \Omega$ is dense in $\mathscr{H}$ with respect to the usual norm, i.e. $D(\delta) \Omega$ is a core for $H$. To prove that $H$ commutes strongly with $\Delta$, note first that by the proof of Lemma 3.3:

$$
(1+i \alpha H) S \xi \doteq S(1+i \alpha H) \xi
$$


for $\xi \in D(\delta) \Omega$ and $\alpha \in \mathbb{R} \backslash\{0\}$. Thus

$$
S(1+i \alpha H)^{-1} \eta=(1+i \alpha H)^{-1} S \eta
$$

for all $\eta \in(1+i \alpha H) D(\delta) \Omega$. But by assumption 1) $(1+i \alpha H) D(\delta) \Omega$ is dense in $D\left(\Delta^{\frac{1}{2}}\right)=D(S)$ with respect to the graph norm of $\Delta^{\frac{1}{2}}$. Hence

$$
S(1+i \alpha H)^{-1} \eta=(1+i \alpha H)^{-1} S \eta \quad \text { for any } \eta \in D\left(\Delta^{\frac{1}{2}}\right)
$$

i.e.

$$
S(1+i \alpha H)^{-1} \supseteqq(1+i \alpha H)^{-1} S \text { for any } \alpha \in \mathbb{R} \backslash\{0\} .
$$

Thus $S$ commutes both with $1+i \alpha H$ and its adjoint $1-i \alpha H$, and hence the components $J$ and $\Delta^{\frac{1}{2}}$ of the polar decomposition of $S$ commute with $(1+i \alpha H)^{-1}$. In particular $H$ commutes strongly with $\Delta$.

\section{§4. A Pathological Example}

Let $\mathscr{M}$ be a von Neumann algebra with a cyclic and separating vector $\Omega, H$ a selfadjoint operator, such that $H \Omega=0$, and $\delta=i[H, \cdot]$ the spatial derivation of $\mathscr{M}$ implemented by $H$. We will show by an example, that essential selfadjointness of $H$ on $D(\delta) \Omega$ is not alone sufficient to ensure that $\delta$ is a generator, and thus the condition that $H$ and $\Delta$ commute strongly is necessary in Theorem 3.1. This settles a conjecture of [3] in the negative. Note that as $\delta\left(A^{*}\right)=\delta(A)^{*}$ we have

$$
\left[i H, J \Delta^{\frac{1}{2}}\right] \xi=0
$$

for any $\xi \in D(\delta) \Omega$, and hence $H$ and $\Delta$ are close to commuting only under the assumption that $D(\delta) \Omega$ is a core for $H$. It is, however, known that two selfadjoint operators may commute on a common invariant core without commuting strongly [18], and similar phenomena can happen in our set-up.

Example 4.1. There exists a von Neumann algebra $\mathscr{M}$ with a separating and cyclic vector $\Omega$, and a selfadjoint operator $H$, such that $H \Omega=0$ and the spatial derivation $\delta=[i H, \cdot]$ of $\mathscr{M}$ implemented by $H$ is $\sigma$-weakly densely defined, and such that $D(\delta) \Omega$ is a core for $H$, but

$$
\exp (i t H) \mathscr{M} \exp (-i t H) \neq \mathscr{M}
$$

for some $t \in \mathbb{R}$. There exists even such an example, where $\mathscr{M}$ is a Type I von Neumann algebra.

Proof. Let $\Omega_{0}$ be a unit vector in a Hilbert space $\mathscr{H}_{0}$, and assume the existence of two von Neumann algebra $\mathscr{N}_{1}$ and $\mathscr{N}_{2}$ on $\mathscr{H}_{0}$, such that $\mathscr{N}_{1} \subseteq \mathscr{N}_{2}, \mathscr{N}_{1} \neq \mathscr{N}_{2}$ and $\Omega_{0}$ is cyclic and separating for both algebras. Examples of this sort occur in quantum field theory, where the Reeh-Schlieder theorem implies the existence of quasi-local algebras with a ground state, which is cyclic and separating for each of the local algebras ([2], Section 24.2). In Example 4.2 we will show that there even exists examples where $\mathscr{N}_{1}$ and $\mathscr{N}_{2}$ are Type I factors. 
Let $\mathbb{T}=\mathbb{R} / \mathbb{Z}$ be the circle group equipped with the normalized Haar measure. Consider the von Neumann algebra $\mathscr{L}\left(\mathscr{H}_{0}\right) \otimes L^{\infty}(\mathbb{T})=L^{\infty}\left(\mathscr{L}\left(\mathscr{H}_{0}\right), \mathbb{T}\right)$ realized as multiplication operators on $\mathscr{H}=L^{2}\left(\mathscr{H}_{0}, \mathbb{T}\right)$. Define an action $\varrho$ of $\mathbb{R}$ on $L^{\infty}\left(\mathscr{L}\left(\mathscr{H}_{0}\right), \mathbb{T}\right)$ by

$$
\left(\varrho_{t} f\right)(s)=f(s-t)
$$

for all $f \in L^{\infty}\left(\mathscr{L}\left(\mathscr{H}_{0}\right), T\right)$. Let $\mathscr{M}$ be the sub von Neumann algebra consisting of those $f \in L^{\infty}(\mathscr{L}(H), \mathbb{T})$, such that

$$
f(s) \in\left\{\begin{array}{lll}
\mathscr{N}_{1} & \text { if } & 0 \leqq S<\frac{1}{2} \\
\mathscr{N}_{2} & \text { if } & \frac{1}{2} \leqq S<1
\end{array}\right.
$$

clearly $\varrho_{t}(\mathscr{M}) \neq \mathscr{M}$ for $t \notin \mathbb{Z}$. Let $\Omega \in \mathscr{H}$ be the vector given by $\Omega(s)=\Omega_{0}$ for all $s \in \mathbb{T}$. Then $\Omega$ is cyclic and separating for $\mathscr{M}$. Moreover $(U(t) \xi)(s)=\xi(s-t), \xi \in L^{2}\left(\mathscr{H}_{0}, \mathbb{T}\right)$ defines a unitary representation of $\mathbb{R}$ on $\mathscr{H}$, such that

$$
\varrho_{t}(f)=U_{t} f U_{t}^{*}
$$

for $f \in L^{\infty}(\mathscr{L}(\mathscr{H}), \mathbb{T})$, and $U_{t} \Omega=\Omega, t \in \mathbb{R}$. Let $\hat{\delta}$ be the infinitesimal generator of $\varrho$ and let $i H$ be the generator of $U_{t}$. Then $D(\hat{\delta})$ consists of the uniformly Hölder continuous functions from $\mathbb{T}$ into $\mathscr{L}\left(\mathscr{H}_{0}\right)$, i.e. the functions $f$, such that $\left\|\varrho_{t} f-f\right\|=0(t)$ as $t \rightarrow 0$. Let $\delta$ be the restriction of $\hat{\delta}$ to

$$
D(\delta)=\left\{f \in L^{\infty}\left(\mathscr{L}\left(\mathscr{H}_{0}\right), \mathbb{T}\right) \mid f \in D(\hat{\delta}) \cap \mathscr{M}, \hat{\delta}(f) \in \mathscr{M}\right\} .
$$

Then $\delta$ is a $\sigma$-weakly densely defined derivation of $\mathscr{M}$, such that $\delta(f)=i[H, f]$ for $f \in \mathscr{M}$. Now $D(\delta) \Omega$ contains the set $\mathscr{D}$ of all elements in $\mathscr{H}$ of the form $s \rightarrow f(s) \Omega_{0}$ where $s \rightarrow f(s)$ is uniformly Hölder continuous and $f(s) \in \mathcal{N}_{1}$ for all $s \in \mathbb{T}$. Hence $\mathscr{D}$ is dense in $\mathscr{H}$, and as $U_{t} \mathscr{D}=\mathscr{D}$ for $t \in \mathbb{R}$, it follows that $\mathscr{D}$ is a core for $H,[15]$. Hence $D(\delta) \Omega$ is a core for $H$. Moreover $H \Omega=0$, but clearly

$$
U_{t} \mathscr{M} U_{t}^{*} \neq \mathscr{M} \text { for } t \notin \mathbb{Z} \text {. }
$$

The last statement of this example follows from Example 4.2:

Example 4.2. There exists two Type I factors $\mathscr{M}_{1}$ and $\mathscr{M}_{2}$ on a separable Hilbert space $\mathscr{H}$, such that $\mathscr{M}_{1} \subseteq \mathscr{M}_{2}, \mathscr{M}_{1} \neq \mathscr{M}_{2}$, and a vector $\Omega \in \mathscr{H}$ such that $\Omega$ is cyclic and separating for both $\mathscr{M}_{1}$ and $\mathscr{M}_{2}$.

Proof. Define $\mathscr{K}=L^{2}(0,2 \pi)$ and $\mathscr{H}=\mathscr{K} \otimes \overline{\mathscr{K}}$ where $\overline{\mathscr{K}}$ is the conjugate Hilbert space of $\mathscr{K}$. Let $P \in \mathscr{L}(\mathscr{K})$ be the orthogonal projection on $L^{2}(0, \pi)$, and define

$$
\begin{aligned}
& \mathscr{N}_{2}=\mathscr{L}(\mathscr{K}) \otimes 1 \\
& \mathscr{N}_{1}=[P \mathscr{L}(\mathscr{K}) P+(1-P) \mathscr{L}(\mathscr{K})(1-P)] \otimes 1 .
\end{aligned}
$$

Clearly $\mathscr{N}_{1} \subseteq \mathscr{N}_{2}, \mathscr{N}_{1} \neq \mathscr{N}_{2}$. Let $\left.r \in\right] 0,1[$, and let $\Omega \in \mathscr{H}$ be the vector defined by

$$
\Omega=\sum_{n=-\infty}^{\infty} r^{|n|} \xi_{n} \otimes \bar{\xi}_{n}
$$

where $\xi_{n}=\frac{1}{\sqrt{2 \pi}} e^{i n s}$. We will show that $\Omega$ is cyclic and separating for both $\mathscr{N}_{1}$ and 
$\mathscr{N}_{2}$. As $\left\{\xi_{n}\right\}_{n \in \mathbb{Z}}$ forms an orthonormal basis for $\mathscr{K}$ it is clear, that $\Omega$ is separating and cyclic for $\mathscr{N}_{2}$, and hence separating for $\mathscr{N}_{1}$. It remains to show that $\Omega$ is cyclic for $\mathscr{N}_{1}$, or equivalently separating for $\mathscr{N}_{1}^{\prime}=P \otimes \mathscr{L}(\overline{\mathscr{K}})+(1-P) \otimes \mathscr{L}(\overline{\mathscr{K}})$. Note that $\mathscr{H}=\mathscr{K} \otimes \overline{\mathscr{K}}$ is isometricly isomorphic with the Hilbert-Schmidt operators on $\mathscr{K}$ by the identification

$$
(\xi \otimes \bar{\eta})(\zeta)=(\eta \mid \zeta) \xi, \quad \xi, \eta, \zeta \in \mathscr{K} .
$$

By this identification $\Omega$ becomes the Hilbert-Schmidt operator with kernel

$$
\begin{aligned}
\Omega_{0}(s, t) & =\frac{1}{2 \pi} \sum_{n=-\infty}^{\infty} r^{|n|} e^{i n(s-t)} \\
& =\frac{1}{2 \pi} \frac{1-r^{2}}{1+r^{2}-2 r \cos (s-t)}
\end{aligned}
$$

which is a real analytic function in $s, t \in[0,2 \pi]$. If $A \in \mathcal{N}_{1}^{\prime}, A=P \otimes \bar{B}+(1-P) \otimes \bar{C}$, where $B, C \in \mathscr{L}(\mathscr{H})$, then $A \Omega$ identifies with the Hilbert-Schmidt operator

$$
P \Omega B+(1-P) \Omega C .
$$

If $A \Omega=0$ it follows by multiplication by $P$ and $1-P$ that

$$
P \Omega B=0, \text { and }(1-P) \Omega C=0 .
$$

But as the kernel $\Omega_{0}(s, t)$ is analytic, it follows that the range $R(\Omega)$ of the HilbertSchmidt operator $\Omega$ consists of analytic functions in $L^{2}(0,2 \pi)$. Hence if $\xi \in L^{2}(0,2 \pi)$ and $P \Omega \xi=0$ then $\Omega \xi=0$, because $\Omega \xi$ is analytic and zero on the interval $[\pi, 2 \pi]$. But as $\Omega$ is injective we have $\xi=0$, i.e. $P \Omega$ is injective.

Therefore $P \Omega B=0$ implies $B=0$. Correspondingly $(1-P) \Omega C=0$ implies $C=0$. Hence if $A \in \mathscr{N}_{1}^{\prime}$ and $A \Omega=0$ it follows that $A=0$, i.e. $\Omega$ is separating for $\mathscr{N}_{1}^{\prime}$. put

Let now $J$ be the modular conjugation associated with the pair $\left(\mathscr{N}_{1}, \Omega\right)$, and

$$
\mathscr{M}_{1}=J \mathscr{N}_{2}^{\prime} J
$$

and

$$
\mathscr{M}_{2}=\mathscr{N}_{2} \text {. }
$$

Clearly $\mathscr{M}_{1} \subseteq J \mathscr{N}_{1}^{\prime} J=\mathscr{N}_{1} \varsubsetneqq \mathscr{N}_{2}=\mathscr{M}_{2}$.

Moreover $\mathscr{M}_{1}$ and $\mathscr{M}_{2}^{\neq}$are Type I factors. Since $\Omega$ is cyclic and separating for $\mathcal{N}_{2}^{\prime}, J \Omega=\Omega$ is cyclic and separating for $J \mathscr{N}_{2}^{\prime} J=\mathscr{M}_{1}$.

Acknowledgements. We are indebted to the following persons for valuable discussions during various stages of this research: M. Pulvirenti, D. W. Robinson, M. Takesaki, and L. Zsido. We would also like to thank CNRS, Marseille and the University of Odense for invitations, which made this collaboration possible. We are grateful to $\mathrm{H}$. Araki for pointing out several minor errors in the preprint version of this paper.

Added Note. We have been informed by Dr. Christian Skau that the conclusion of Example 4.2 ist due to Kadison [29]. The result follows also very easily from Dixmier and Marechal [30]. 


\section{References}

1. Arveson,W.: On groups of automorphisms of operator algebras. J. Funct. Anal. 15, 217-243 (1974)

2. Bogolubov, N. N., Lagunov, A. A., Todorov, I. T.: Introduction to axiomatic quantum field theory. Reading, Mass.: Benjamin 1975

3. Bratteli,O., Robinson,D.W.: Unbounded derivations of von Neumann algebras. Ann. Inst. Henri Poincaré A 25, 139-164 (1976)

4. Bratteli,O., Robinson,D.W.: Unbounded derivations and invariant trace states. Commun. math. Phys. 46, 31-35 (1976)

5. Bratteli,O., Robinson,D.W.: Greens functions, Hamiltonians, and modular automorphisms. Commun. math. Phys. 50, 135-156 (1976)

6. Bratteli,O., Herman, R.H., Robinson,D.W.: Quasianalytic vectors and derivations of operator algebras. Math. Scand. 39, 371-381 (1976)

7. Bratteli,O.: Unbounded derivations and invariant states. ZIF Preprint, unpublished (1976)

8. Connes, A.: Une classification de facteurs de type III. Ann. Sci. Ecole Norm. Sup. 6, 133-252 (1973)

9. Digernes, T.: Duality for weights on covariant systems and its application. U.C.L.A. Thesis (1975)

10. Gallavotti,G., Pulvirenti,M.: Classical KMS condition and Tomita-Takesaki theory. Commun. math. Phys. 46, 1-9 (1976)

11. Greenleaf,F.P.: Invariant means on topological groups. New York: van Nostrand 1969

12. Haag, R., Kastler,D.: An algebraic approach to quantum field theory. J. Math. Phys. 5, 848-861 (1964)

13. Haagerup, U.: On the dual weights for von Neumann algebras. I. Preprint, Odense (1975)

14. Herman, R. H. : Implementation and the core problem. ZIF Preprint (1975)

15. Kato, T.: Perturbation theory for linear operators. Berlin-Heidelberg-New York: Springer 1966

16. Leeuw,K. de: On the adjoint semigroup and some problems in the theory of approximation. Math. Z. 73, 219-239 (1960)

17. Nelson,E.: Notes on non-commutative integration. J. Funct. Anal. 15, 103-116 (1974)

18. Nelson, E.: Analytic vectors. Ann. Math. 70, 572-615 (1959)

19. Pedersen,G.K., Takesaki,M.: The Radon-Nikodym theorem for von Neumann algebras. Acta Math. 130, 53-87 (1973)

20. Pulvirenti,M., Tirozzi,B.: Time evolution of a quantum lattice system. Commun. math. Phys. 30 , 83-98 (1973)

21. Robinson,D.W.: Statistical mechanics for quantum spin systems. Commun. math. Phys. 7, 337348 (1968)

22. Robinson,D.W.: Dynamics in quantum statistical mechanics. Proceedings of the ZIF Conference, Bielefeld 1976 (to appear)

23. Segal,I.: A non-commutative extention of abstract integration. Ann. Math. 58, 595-596 (1953)

24. Streater, R.: On certain non-relativistic quantized fields. Commun. math. Phys. 7, 93-98 (1968)

25. Takesaki,M.: Tomitas theory of modular Hilbert algebras and its applications. Lecture notes in mathematics, Vol. 128. Berlin-Heidelberg-New York: Springer 1970

26. Takesaki, M.: The structure of a von Neumann algebra with a homogenuous periodic state. Acta Math. 131, 79-121 (1973)

27. Takesaki,M.: Duality for crossed products and the structure of von Neumann algebras of type III. Acta Math. 131, 249-310 (1973)

28. Winnink,M.: In: Statistical mechanics and field theory (eds. R. Sen, C. Weil). Jerusalem: Keter Publishing House 1971

29. Kadison, R.: Remarks on the type of von Neumann algebras of local observables in quantum field theory. J. Math. Phys. 4, 1511-1516 (1963)

30. Dixmier,J., Marechal,O.: Vecteurs totalisateurs d'une algèbre de von Neumann. Commun. math. Phys. 22, 44-50 (1970) 
